\title{
The coarse Baum-Connes conjecture for Busemann nonpositively curved spaces
}

\author{
Tomohiro Fukaya and Shin-ichi Oguni
}

\begin{abstract}
We prove that the coarse assembly maps for proper metric spaces that are nonpositively curved in the sense of Busemann are isomorphisms, where we do not assume that the spaces have bounded coarse geometry. Also it is shown that we can calculate the coarse $K$-homology and the $K$-theory of the Roe algebra by using the visual boundaries.
\end{abstract}

\section{Introduction}

For a proper metric space $X$, the coarse assembly map

$$
\mu(X): K X_{*}(X) \rightarrow K_{*}\left(C^{*} X\right)
$$

is defined as a coarse index map (see [3], [7], [11]). Here $K X_{*}(X)$ is the coarse $K$-homology of $X$ and $K_{*}\left(C^{*} X\right)$ is the $K$-theory of the Roe algebra $C^{*} X$. It is well known that $K X_{*}(X), K_{*}\left(C^{*} X\right)$, and $\mu(X)$ depend only on the large-scale geometry of $X$, that is, the coarse structure given by the metric on $X$ (refer to $[3$, Section 6$]$ ). It would be interesting to know which proper metric space $X$ satisfies the property that $\mu(X)$ is an isomorphism (resp., a rational injection) because the property is closely related to the analytic Novikov conjecture (resp., nonpermission of uniformly positive scalar curvatures). In particular, the following is called the coarse Baum-Connes conjecture: if a proper metric space $X$ is reasonable, for example, uniformly contractible and with bounded coarse geometry, then $\mu(X)$ is an isomorphism (see the Appendix for more details on bounded coarse geometry). Now we can recognize many spaces whose coarse assembly maps are isomorphisms. For example, if $X$ has bounded coarse geometry and is coarsely embeddable into a Hilbert space, then $\mu(X)$ is an isomorphism (see [12, Theorem 1.1]). Also Higson and Roe [3, Corollary 8.2] showed that if $X$ is geodesic and hyperbolic in the sense of Gromov, then $\mu(X)$ is an isomorphism, where bounded coarse geometry is not assumed.

Kyoto Journal of Mathematics, Vol. 56, No. 1 (2016), 1-12

DOI $10.1215 / 21562261-3445129$, (C) 2016 by Kyoto University

Received October 14, 2014. Revised October 16, 2014. Accepted October 16, 2014.

2010 Mathematics Subject Classification: 58J22.

Fukaya's work supported by Grant-in-Aid for Scientific Research for Young Scientists (B) (No. 23740049) from the Japan Society for the Promotion of Science.

Oguni's work supported by Grant-in-Aid for Scientific Research for Young Scientists (B) (No. 24740045) from the Japan Society for the Promotion of Science. 
Now we consider which proper metric space $X$ has a boundary with information about $K X_{*}(X)$ and $K_{*}\left(C^{*} X\right)$. This question is a variant of the Weinberger conjecture, which gives a sufficient condition for the property that the assembly map is rationally injective (see [7, Conjecture 6.33 and Proposition 6.34]). Let $W$ be a boundary which gives a coarse compactification of a proper metric space $X$ (see Section 2). Then we have the following commutative diagram:

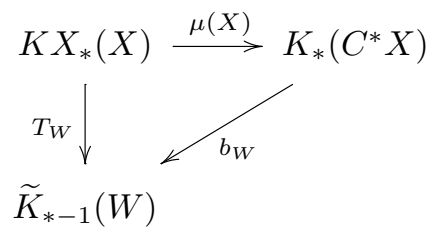

Here $\widetilde{K}_{*-1}(W)$ is the reduced $K$-homology of $W, T_{W}$ is a transgression map, and $b_{W}$ is a map defined in [3, Appendix] (refer to [2, Section 1.2]). There are some classes of metric spaces $X$ admitting coarse compactifications $X \cup W$ such that $T_{W}$ and $b_{W}$ are isomorphisms. Such a typical class consists of unbounded proper geodesic hyperbolic spaces in the sense of Gromov (see [2, Corollary 5.3]). Indeed, the Gromov completions are desired coarse compactifications. See [2] for other classes.

In this note, we deal with proper metric spaces which are nonpositively curved in the sense of Busemann (for short, proper Busemann spaces). Proper CAT(0)-spaces are typical examples. The following is our main theorem.

\section{THEOREM 1.1}

Let $X$ be an unbounded proper Busemann space (which does not necessarily have bounded coarse geometry), and let $\partial_{v} X$ be the visual boundary. Then $\mu(X)$ is an isomorphism. Also $X \cup \partial_{v} X$ is a coarse compactification of $X$, and moreover $T_{\partial_{v} X}$ and $b_{\partial_{v} X}$ are isomorphisms.

\section{REMARK 1.2}

A key in our proof of Theorem 1.1 is to show that the coarsening map

$$
c(X): K_{*}(X) \rightarrow K X_{*}(X)
$$

is an isomorphism for any proper Busemann space $X$. In general, when a proper metric space is uniformly contractible and has bounded coarse geometry, the coarsening map is an isomorphism (see [3, Proposition 3.8], [1, proof of Theorem 4.8], [2, Section 3.2]). Proper Busemann spaces are uniformly contractible, but they do not necessarily have bounded coarse geometry (see Example A.5). Higson and Roe [3, below Corollary 7.5] claimed that a proper nonpositively curved space $X$ without necessarily bounded coarse geometry satisfies that the coarsening map is an isomorphism, but they did not give details. Indeed, we prove it for proper Busemann spaces (see Proposition 3.4). 


\section{The visual boundaries of proper Busemann spaces}

In this section, we study the visual boundaries of proper Busemann spaces from a coarsely geometric viewpoint. We refer to [9], which deals with CAT(0)-spaces (see also [10]).

The following is one of the equivalent definitions of Busemann spaces (see [6, Proposition 8.1.2(viii)]).

\section{DEFINITION 2.1}

Let $X$ be a space endowed with a metric $d$. We call $X$ a nonpositively curved space in the sense of Busemann (for short, a Busemann space) if $X$ is geodesic and satisfies the following: for any points $x_{0}, x_{1}, y_{0}, y_{1} \in X$, any geodesics $\overline{x_{0} x_{1}}, \overline{y_{0} y_{1}}$, and any $t \in[0,1], d\left(x_{t}, y_{t}\right)$ is smaller than or equal to $(1-t) d\left(x_{0}, y_{0}\right)+t d\left(x_{1}, y_{1}\right)$, where $x_{t} \in \overline{x_{0} x_{1}}$ and $y_{t} \in \overline{y_{0} y_{1}}$ with $d\left(x_{0}, x_{t}\right)=t d\left(x_{0}, x_{1}\right)$ and $d\left(y_{0}, y_{t}\right)=t d\left(y_{0}, y_{1}\right)$, respectively.

Typical examples of Busemann nonpositively curved spaces include CAT(0)spaces and strictly convex Banach spaces like $l^{p}$-spaces $(1<p<\infty$; see also Example 2.2).

We consider the visual boundaries. Let $X$ be a proper Busemann space. Fix a base point $o \in X$. Since two points in a Busemann space are connected by a unique geodesic, we have a continuous map

$$
X \times[0,1] \ni(x, t) \mapsto \delta_{t}(x) \in X,
$$

where $\delta_{t}(x)$ is characterized as a point on a geodesic from $o$ to $x$ with $d\left(o, \delta_{t}(x)\right)=$ $t d(o, x)$. For each $t \in(0, \infty)$, put $B(o, t):=\{a \in X \mid d(o, a) \leq t\}$. For any $s, t \in$ $(0, \infty)$ with $s<t$, we define a surjection $\pi_{s, t}: B(o, t) \rightarrow B(o, s)$ as $\pi_{s, t}(a):=a$ if $d(o, a) \leq s$ and $\pi_{s, t}(a):=\delta_{s / d(o, a)}(a)$ if $d(o, a)>s$. Also for any $t \in(0, \infty)$, we define a surjection $\pi_{t}: X \rightarrow B(o, t)$ as $\pi_{t}(a):=a$ if $d(o, a) \leq t$ and $\pi_{t}(a):=$ $\delta_{t / d(o, a)}(a)$ if $d(o, a)>t$. We consider a projective system consisting of $\left\{\pi_{s, t}\right.$ : $B(o, t) \rightarrow B(o, s)\}_{0<s<t}$. Then the projective $\operatorname{limit}_{\leftarrow} \lim _{\leftarrow} B(o, t)$ contains $X$ as an open dense subset by the map $\lim _{\leftarrow} \pi_{t}: X \rightarrow \lim _{\leftarrow} B(o, t)$. We put $\bar{X}:=$ $\lim _{\leftarrow} B(o, t)$ and $\partial_{v} X:=\bar{X} \backslash X$. We call $\partial_{v} X$ the visual boundary of $X$. The visual boundary of $X$ is independent (up to canonical homeomorphisms) of the choice of base points by [5, Main Theorem].

\section{EXAMPLE 2.2}

Let $p$ belong to $(1, \infty)$. For each positive integer $n$, denote by $\ell_{p}(n)$ the $n$ dimensional $\ell_{p}$-space. Then $\ell_{p}(n)$ is a proper Busemann space and the visual boundary is homeomorphic to the $(n-1)$-dimensional sphere $S^{n-1}$. We remark that $\ell_{p}(n)$ is $\operatorname{CAT}(0)$ if and only if $n=1$ or $p=2$.

For each $p \in(1, \infty)$, we give an example which is a proper Busemann space without bounded coarse geometry. First we consider a half-line $[0, \infty)$ with the standard metric by absolute values. Next we identify the zero vector of $\ell_{p}(n)$ with $n \in[0, \infty)$ for all positive integers $n$. Finally we endow the resulting space $X_{p}$ with 
the path metric, where the embeddings $\ell_{p}(n) \rightarrow X_{p}$ for all $n \in \mathbb{N}$ and $[0, \infty) \rightarrow X_{p}$ are isometric. Then $X_{p}$ is a proper Busemann space and the visual boundary of $X_{p}$ is homeomorphic to the one-point compactification of $\bigsqcup_{n \in \mathbb{N}} \partial_{v} \ell_{p}(n)$, that is, the one-point compactification of $\bigsqcup_{n \in \mathbb{N}} S^{n-1}$. Obviously $X_{p}$ is $\operatorname{CAT}(0)$ if and only if $p=2$. In the Appendix we show that $X_{p}$ does not have bounded coarse geometry.

The following is implied by the argument in [9, proof of Lemma 4.6.1] for proper CAT(0)-spaces and the visual boundaries.

PROPOSITION 2.3

Let $X$ be a proper Busemann space. Then $X \cup \partial_{v} X$ is contractible.

Now we recall a definition of coarse compactifications. Let $X$ be a proper metric space. We denote by $C_{b}(X)$ the set of all bounded continuous functions of $X$. An element $f \in C_{b}(X)$ is a Higson function if, for any $\epsilon>0$ and any $R>0$, there exists a bounded set $K \subset X$ such that $x, y \in X$ with $d(x, y)<R$ and $x, y \notin K$ satisfy $|f(x)-f(y)|<\epsilon$. We denote by $C_{h}(X)$ the set of all Higson functions of $X$. Let $\bar{X}$ be a compact metrizable space which is a compactification of $X$. We denote by $C(\bar{X})$ the set of all continuous functions of $\bar{X}$. Then $C(\bar{X})$ is naturally identified with a subalgebra of $C_{b}(X)$ by the Gelfand-Naimark theorem. After the identification, if every element of $C(\bar{X})$ is a Higson function, then we call $\bar{X}$ a coarse compactification of $X$.

The following is known for proper CAT(0)-spaces and the visual boundaries (see [9, Lemma 4.6.2]).

\section{PROPOSITION 2.4}

Let $X$ be a proper Busemann space. Then $X \cup \partial_{v} X$ is a coarse compactification of $X$.

\section{Proof}

We need to modify the argument in [9, proof of Lemma 4.6.2] because that proof uses the $\operatorname{CAT}(0)$-inequality.

We recall that $X \cup \partial_{v} X=\lim _{\leftarrow} B(o, t)$. We naturally regard $C(B(o, t))$ as a subset of $C_{b}(X)$ by

$$
C(B(o, t)) \ni f \mapsto f \circ \pi_{t} \in C_{b}(X),
$$

where $C(B(o, t))$ is the set of all continuous functions on $B(o, t)$. Then $C(X \cup$ $\left.\partial_{v} X\right)$ is identified with the closure of $\bigcup_{t>0} C(B(o, t))$. We take $t \in(0, \infty)$ and $f \in C(B(o, t))$. Then it is enough to prove that $F:=f \circ \pi_{t}$ is a Higson function of $X$. We take $\epsilon>0$ and $R>0$. Since $B(o, t+R)$ is compact, we have $\delta>0$ such that $\left|F\left(a^{\prime}\right)-F\left(b^{\prime}\right)\right|<\epsilon$ for any $a^{\prime}, b^{\prime} \in B(o, t+R)$ with $d\left(a^{\prime}, b^{\prime}\right)<\delta$. We take any $a, b \in X$ with $d(a, b)<R$ and we have

$$
r:=\min \left\{r_{a}:=d(o, a), r_{b}:=d(o, b)\right\}>S:=\max \{t, t R / \delta\} .
$$


We put $a^{\prime}=\delta_{t / r}(a)$ and $b^{\prime}=\delta_{t / r}(b)$. Then we have

$$
t \leq d\left(o, a^{\prime}\right)=t r_{a} / r<t+R, \quad t \leq d\left(o, b^{\prime}\right)=t r_{b} / r<t+R,
$$

$F\left(a^{\prime}\right)=F(a)$, and $F\left(b^{\prime}\right)=F(b)$. Since $X$ is a Busemann space, we have

$$
d\left(a^{\prime}, b^{\prime}\right)=d\left(\delta_{t / r}(a), \delta_{t / r}(b)\right) \leq \frac{t}{r} d(a, b)<\frac{t R}{r} \leq \delta .
$$

Hence we have $|F(a)-F(b)|<\epsilon$.

\section{The coarse assembly maps for proper Busemann spaces}

In this section we prove that the coarse assembly maps for proper Busemann spaces are isomorphisms. Let $X$ be a proper metric space. Then we have the following commutative diagram:

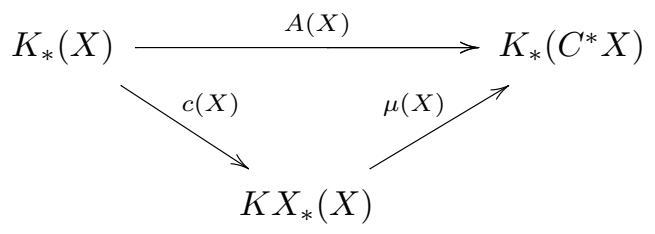

Here $K_{*}(X)$ is the $K$-homology of $X, C^{*} X$ is the Roe algebra of $X, K_{*}\left(C^{*} X\right)$ is the $K$-theory of $C^{*} X, A(X)$ is the assembly map for $X, c(X)$ is the coarsening map for $X$, and $\mu(X)$ is the coarse assembly map for $X$ (see [3, Section 6] and also [2, Section 1]).

If a proper metric space $X$ is a scalable space (which does not necessarily have bounded coarse geometry), then Higson and Roe proved that the assembly map $A(X)$ is an isomorphism by a so-called Eilenberg swindle (see [3, Theorem 7.2], [4, Theorem 12.4.11]). Proper CAT(0)-spaces are such examples (see [4, Exercise 12.7.4], [9, proof of Proposition 4.6.3]). Their proof works well for proper Busemann spaces and thus we have the following result.

\section{PROPOSITION 3.1}

Let $X$ be a proper Busemann space. Then $X$ is scalable and thus $A(X)$ is an isomorphism.

We recall definitions of coarse maps. Let $\left(X, d_{X}\right)$ and $\left(Y, d_{Y}\right)$ be proper metric spaces. A map $f: Y \rightarrow X$ is a coarse map if $f$ satisfies the following:

- for any bounded subset $K$ of $X, f^{-1}(K)$ is bounded;

- for any $R>0$, there exists $S>0$ such that $y, y^{\prime} \in Y$ with $d_{Y}\left(y, y^{\prime}\right)<R$ satisfy $d_{X}\left(f(y), f\left(y^{\prime}\right)\right)<S$.

Two coarse maps $f, g: Y \rightarrow X$ are close if there exists $C>0$ such that $d_{X}(f(y)$, $g(y))<C$ for any $y \in Y$.

We need two lemmas to prove Proposition 3.4. 


\section{LEMMA 3.2}

Let $X$ be a proper Busemann space, let $Y$ be a connected locally finite simplicial complex with the spherical metric, and let $f: Y \rightarrow X$ be a coarse map. Then there exists a continuous coarse map $g: Y \rightarrow X$ such that $g$ and $f$ are close.

Here we briefly explain the spherical metric. Let $Y$ be a connected locally finite simplicial complex. Denote the 0 th skeleton by $Y^{(0)}:=\left\{v_{i}\right\}_{i \in \mathbb{N}}$. Then for every nonnegative integer $k$, we identify every $k$-simplex with vertices $\left\{v_{i_{1}}, \ldots, v_{i_{k+1}}\right\}$ of $Y$ with

$$
\Delta_{S}^{k}:=\left\{\left(u_{i_{1}}, \ldots, u_{i_{k+1}}\right) \in[0,1]^{k+1} \mid \sum_{j=1}^{k+1} u_{i_{j}}^{2}=1\right\} .
$$

By using the identification, we have so-called spherical coordinates $\left\{u_{i}: Y \rightarrow\right.$ $[0,1]\}_{i \in \mathbb{N}}$, where $\sum_{i \in \mathbb{N}} u_{i}(y)^{2}$ is a finite sum and equal to 1 . Now we have an embedding $Y \ni y \mapsto\left(u_{1}(y), u_{2}(y), \ldots\right) \in S\left(\ell_{2}(\mathbb{N})\right)$, where $S\left(\ell_{2}(\mathbb{N})\right)$ is the sphere with radius 1 in $\ell_{2}(\mathbb{N})$. We endow $Y$ with the metric $d_{S}$ induced from the path metric of the image of the embedding and call $d_{S}$ the spherical metric. The spherical metric is very familiar with the graph metric in the following sense: the restriction of $d_{S}$ on the first skeleton $Y^{(1)}$ is equal to the graph metric on $Y^{(1)}$ up to $\pi / 2$-multiplication.

\section{Proof of Lemma 3.2}

We denote by $Y^{(0)}$ the 0 th skeleton of $Y$. Then we construct a continuous coarse map $g: Y \rightarrow X$ with $\left.g\right|_{Y^{(0)}}=\left.f\right|_{Y^{(0)}}$ such that $g$ and $f$ are close.

Every point $y$ of $Y$ is uniquely presented by the barycentric coordinate $\left\{\left(v_{1}, t_{1}\right), \ldots,\left(v_{n}, t_{n}\right)\right\}$, where $v_{i} \in Y^{(0)}$ and $t_{i} \in(0,1]$ with $\sum_{i=1}^{n} t_{i}=1$. Now for such a point $y$, we define $g(y) \in X$ as the barycenter of $\left\{\left(f\left(v_{1}\right), t_{1}\right), \ldots\right.$, $\left.\left(f\left(v_{n}\right), t_{n}\right)\right\}$; that is, $g(y)$ is a unique point which attains the minimum of (see [6, Definition 8.4.10])

$$
X \ni x \mapsto \sum_{i=1}^{n} t_{i} d_{X}\left(x, f\left(v_{i}\right)\right)^{2} \in[0, \infty) .
$$

Then we have a map $g: Y \rightarrow X$ with $\left.g\right|_{Y^{(0)}}=\left.f\right|_{Y^{(0)}}$. Since the barycenter continuously depends on weights $t_{i}$, the map $g$ is continuous.

Now we prove that $g$ is close to $f$. Since $f$ is coarse, we have $C>0$ such that, for any point $y, y^{\prime} \in Y$ contained in the same simplex, $d_{X}\left(f(y), f\left(y^{\prime}\right)\right)<C$. Take a point $y \in Y$ which is presented by the barycentric coordinate $\left\{\left(v_{1}, t_{1}\right), \ldots\right.$, $\left.\left(v_{n}, t_{n}\right)\right\}$. Then we have $i_{0} \in\{1, \ldots, n\}$ satisfying $d\left(g(y), f\left(v_{i_{0}}\right)\right) \leq d\left(g(y), f\left(v_{i}\right)\right)$ for any $i \in\{1, \ldots, n\}$. Since $g(y)$ is the barycenter of $\left\{\left(f\left(v_{1}\right), t_{1}\right), \ldots,\left(f\left(v_{n}\right), t_{n}\right)\right\}$ with $\sum_{i=1}^{n} t_{i}=1$, we have

$$
d\left(g(y), f\left(v_{i_{0}}\right)\right)^{2}=\sum_{i=1}^{n} t_{i} d\left(g(y), f\left(v_{i_{0}}\right)\right)^{2} \leq \sum_{i=1}^{n} t_{i} d\left(g(y), f\left(v_{i}\right)\right)^{2}
$$




$$
<\sum_{i=1}^{n} t_{i} d\left(f\left(v_{i_{0}}\right), f\left(v_{i}\right)\right)^{2} \leq \sum_{i=1}^{n} t_{i} C^{2}=C^{2} .
$$

Since we have $d\left(f(y), f\left(v_{i_{0}}\right)\right)<C$ and $f\left(v_{i_{0}}\right)=g\left(v_{i_{0}}\right)$, the triangle inequality implies $d(g(y), f(y))<2 C$. Since $g$ is close to the coarse map $f, g$ is also a coarse map.

\section{LEMMA 3.3}

Let $X$ be a proper Busemann space, and let $\phi: X \rightarrow X$ be a continuous coarse map which is close to $\mathrm{id}_{X}$. Then $\mathrm{id}_{X}$ and $\phi$ are properly homotopic.

Proof

We consider the map

$$
h: X \times[0,1] \ni(x, t) \mapsto h(x, t) \in X,
$$

where $h(x, t)$ is characterized as a point on the geodesic from $x$ to $\phi(x)$ with $d(x, h(x, t))=t d(x, \phi(x))$. Then $h$ is continuous in view of Definition 2.1. Hence, $h$ is a proper homotopy between $\operatorname{id}_{X}$ and $\phi$.

\section{PROPOSITION 3.4}

Let $X$ be a proper Busemann space. Then $c(X)$ is an isomorphism.

\section{Proof}

The argument in [3, proof of Proposition 3.8] works well in our situation when we use Lemmas 3.2 and 3.3 instead of [3, Lemmas 3.3 and 3.4]. We give a sketch for the reader's convenience. First of all, we take an anti-Čech system $\mathcal{U}_{1}, \mathcal{U}_{2}, \ldots$ of $X$ and a partition of unity of $\mathcal{U}_{1}$. When we denote by $\left|\mathcal{U}_{i}\right|$ the geometrization of $\mathcal{U}_{i}$ as a nerve complex for each $i \in \mathbb{N}$, we have a coarsening sequence

$$
X \stackrel{\varphi}{\longrightarrow}\left|\mathcal{U}_{1}\right| \stackrel{\varphi_{1}}{\longrightarrow}\left|\mathcal{U}_{2}\right| \stackrel{\varphi_{2}}{\longrightarrow} \cdots,
$$

where all maps are proper continuous maps and coarse equivalences. By Lemma 3.2, we have a continuous coarse map $g_{i}:\left|\mathcal{U}_{i}\right| \rightarrow X$ for each $i \in \mathbb{N}$ satisfying that $g_{i} \circ\left(\varphi_{i-1} \circ \cdots \circ \varphi_{1} \circ \varphi\right)$ and $\left(\varphi_{i-1} \circ \cdots \circ \varphi_{1} \circ \varphi\right) \circ g_{i}$ are close to $\operatorname{id}_{X}$ and $\operatorname{id}_{\left|\mathcal{U}_{i}\right|}$, respectively. Then Lemma 3.3 implies that $g_{i} \circ\left(\varphi_{i-1} \circ \cdots \circ \varphi_{1} \circ \varphi\right)$ and $\operatorname{id}_{X}$ are properly homotopic. Since $\left(\varphi_{i-1} \circ \cdots \circ \varphi_{1} \circ \varphi\right) \circ g_{i}$ and $\operatorname{id}_{\left|\mathcal{U}_{i}\right|}$ are close, we have $k \geq i$ such that $\left(\varphi_{k} \circ \cdots \circ \varphi_{1} \circ \varphi\right) \circ g_{i}$ and $\varphi_{k} \circ \cdots \circ \varphi_{i}$ are contiguous and thus properly homotopic. Then the coarsening sequence implies that $K_{*}(X) \cong$ $\stackrel{\lim }{\longrightarrow} K_{*}\left(\left|\mathcal{U}_{i}\right|\right)$, where the right-hand side is $K X_{*}(X)$ by definition.

Propositions 3.1 and 3.4 imply that $\mu(X)$ is an isomorphism for a proper Busemann space (see the commutative diagram (1)).

\section{Proof of Theorem 1.1}

In this section we complete a proof of Theorem 1.1. 
When $X$ is an unbounded proper metric space and $X \cup W$ is a coarse compactification of $X$, we have the following commutative diagram:

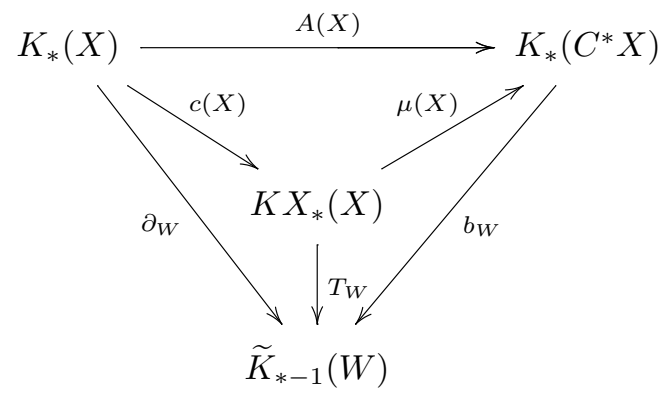

where $\partial_{W}$ is a boundary map of the homological long exact sequence for a pair $(X \cup W, W)$ and $b_{W}$ is a map defined in [3, Appendix] (see also [2, Section 1.2]).

Proof of Theorem 1.1

Let $X$ be an unbounded proper Busemann space, and consider the visual boundary $\partial_{v} X$. We already proved that $A(x), c(X)$, and $\mu(X)$ are isomorphisms in Section 3. Since $X \cup \partial_{v} X$ is contractible by Proposition 2.3, $\partial_{\partial_{v} X}$ is an isomorphism. Now the assertion follows from the commutative diagram above.

EXAMPLE 4.1

Let $p$ belong to $(1, \infty)$. We consider $X_{p}$ in Example 2.2. Then Theorem 1.1 implies the following:

$$
\begin{aligned}
K X_{*}\left(X_{p}\right) & \cong K_{*}\left(C^{*} X_{p}\right) \cong \widetilde{K}_{*-1}\left(\partial_{v} X_{p}\right) \\
& \cong K_{*-1}\left(\bigsqcup_{n \in \mathbb{N}} S^{n-1}\right) \cong \prod_{n \in \mathbb{N}} K_{*-1}\left(S^{n-1}\right) \cong \prod_{n \in \mathbb{N}} \mathbb{Z},
\end{aligned}
$$

where we used the strong excision property and the cluster axiom (see [4, Definition 7.3.1]).

\section{Coarse $K$-theories and coarse cohomologies of proper Busemann spaces}

In this section we briefly consider coarse $K$-theories and coarse cohomologies of proper Busemann spaces. Let $X$ be a proper metric space, and let $X \cup W$ be a coarse compactification of $X$. Then we have the following commutative diagram:

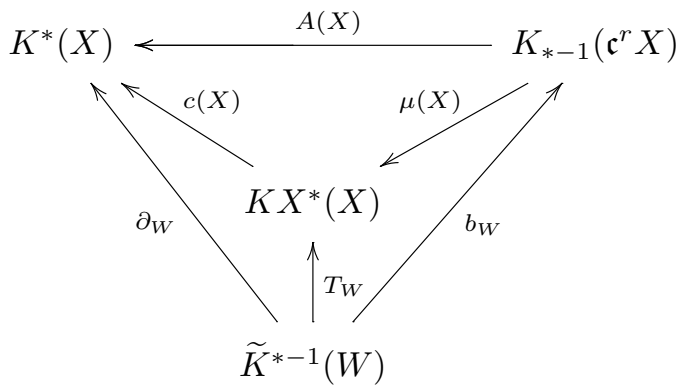


Here $K^{*}(X), \mathfrak{c}^{r} X, K_{*}\left(\mathfrak{c}^{r} X\right), K X^{*}(X), \widetilde{K}^{*}(W), A(X), \mu(X), c(X), \partial_{W}, b_{W}$, and $T_{W}$ are the $K$-theory of $X$, the reduced stable Higson corona of $X$, the $K$-theory of $\mathfrak{c}^{r} X$, the coarse $K$-theory of $X$, the reduced $K$-theory of $W$, the co-assembly map of $X$, the coarse co-assembly map of $X$, the character map of $X$, the boundary map of the cohomological long exact sequence of $(X \cup W, W)$, the map induced by the inclusion of $C(W)$ into $\mathfrak{c}^{r} X$, and the transgression map, respectively (see [1, Section 4], [9, Chapter 4], [2, Sections 3, 4] for details).

When we consider a proper Busemann space $X$ and the visual boundary $\partial_{v} X$, we have the following by a similar argument to that in the proof of Theorem 1.1, but we omit details.

\section{THEOREM 5.1}

Let $X$ be an unbounded proper Busemann space (which does not necessarily have bounded coarse geometry), and let $\partial_{v} X$ be the visual boundary. Then in the above commutative diagram, all maps $A(X), c(X), \mu(X), \partial_{\partial_{v} X}, b_{\partial_{v} X}$, and $T_{\partial_{v} X}$ are isomorphisms.

If $X$ is an unbounded proper CAT(0)-space with bounded coarse geometry, then the above is already known (see [1, Theorem 4.8], [9, Section 4.6]).

Similarly we have the following result.

\section{THEOREM 5.2}

Let $X$ be an unbounded proper Busemann space (which does not necessarily have bounded coarse geometry), and let $\partial_{v} X$ be the visual boundary. Then all maps in the commutative diagram

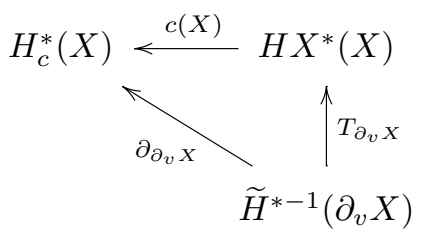

are isomorphisms. Here $H_{c}^{*}(X), H X^{*}(X), \widetilde{H}^{*}(W), c(X), \partial_{W}$, and $T_{W}$ are the compactly supported Alexander-Spanier cohomology of $X$, the coarse cohomology of $X$, the reduced Alexander-Spanier cohomology of $W$, the character map of $X$, the boundary map of the cohomological long exact sequence of $(X \cup W, W)$, and the transgression map, respectively (see [7], [2, Section 3] for details).

In the above statement, we can show that $c(X)$ is an isomorphism in a different way. Indeed, it is known that the compactly supported Alexander-Spanier cohomology and the coarse cohomology are isomorphic by the character map for any uniformly contractible proper metric space without necessarily bounded coarse geometry (see [7, Proposition 3.33]). We note that Busemann spaces are uniformly contractible. 


\section{Appendix: Bounded coarse geometry}

In this appendix we collect some equivalent definitions of bounded coarse geometry for the reader's convenience (see also [8, Section 3.1]) and prove that $X_{p}$ in Example 2.2 does not have bounded coarse geometry.

\section{DEFINITION A.1}

Let $\Gamma$ be a discrete metric space. Then $\Gamma$ is said to have bounded geometry if, for any $R>0$,

$$
\sup \{\# B(\gamma, R) \mid \gamma \in \Gamma\}<\infty
$$

When a discrete metric space has bounded geometry, so does every subset with the restricted metric.

The following was introduced by Fan (see [3, Definition 3.6], [8, Definition 3.9]).

\section{DEFINITION A.2}

Let $X$ be a metric space. Then $X$ is said to have bounded coarse geometry if there exists $\epsilon>0$ satisfying the following: for any $R>0$,

$$
\sup \left\{l \mid x \in X, x_{1}, \ldots, x_{l} \in B(x, R), i \neq j, d\left(x_{i}, x_{j}\right)>\epsilon\right\}<\infty .
$$

When a metric space has bounded coarse geometry, so does every subset with the restricted metric.

Bounded coarse geometry is simply called bounded geometry in much of the literature. However, a discrete metric space with bounded coarse geometry does not necessarily have bounded geometry in the sense of Definition A.1. Therefore, we adopt the notion of bounded coarse geometry.

\section{DEFINITION A.3}

Let $X$ be a metric space, and let $\Gamma$ be a discrete subset of $X$. For $\epsilon>0$ and $C \geq 0, \Gamma$ is said to be $\epsilon$-separated if every pair of two distinct elements $\gamma_{1}, \gamma_{2} \in \Gamma$ satisfies $d\left(\gamma_{1}, \gamma_{2}\right)>\epsilon$. For $C \geq 0, \Gamma$ is said to be $C$-dense if every $x \in X$ satisfies $d(x, \gamma) \leq C$ for some $\gamma \in \Gamma$. Also $\Gamma$ is called a net of $X$ if $\Gamma$ is $C$-dense for some $C \geq 0$.

We can show that every metric space has an $\epsilon$-separated net for any $\epsilon>0$ by using Zorn's lemma.

PROPOSITION A.4

Let $X$ be a metric space. The following are equivalent.

(a) X has bounded coarse geometry.

(b) $X$ has a net with bounded geometry. 
(c) $X$ is coarsely equivalent to a discrete metric space with bounded geometry.

(d) There exists $\epsilon>0$ such that every $\epsilon^{\prime}$-net of $X$ for any $\epsilon^{\prime} \geq \epsilon$ has bounded geometry.

(e) There exists $\epsilon>0$ satisfying the following: for any $R>0$, there exists $N>0$ such that, for any $x \in X$,

$$
B(x, R) \subset \bigcup_{i=1, \ldots, N} B\left(x_{i}, \epsilon\right)
$$

for some $x_{1}, \ldots, x_{N} \in X$.

\section{Proof}

Conditions (a) and (e) are equivalent by [8, Proposition 3.2(d)]. It is easy to show equivalence between conditions (b) and (c). Trivially, condition (d) implies condition (b).

Now we prove that condition (a) implies condition (d). Take $\epsilon$ in Definition A.2. We consider any $\epsilon^{\prime}$-separated net $\Gamma$ where $\epsilon^{\prime} \geq \epsilon$. Then for any $R>0$ and any $\gamma \in \Gamma$,

$$
\begin{aligned}
& \#(B(\gamma, R) \cap \Gamma) \\
& \quad \leq \sup \left\{l \mid x \in X, x_{1}, \ldots, x_{l} \in B(x, R), i \neq j, d\left(x_{i}, x_{j}\right)>\epsilon\right\} .
\end{aligned}
$$

Hence, $\Gamma$ has bounded geometry.

Finally we show that condition (b) implies condition (e). Suppose that $\Gamma$ has bounded geometry and is a $C$-dense net of $X$ for some $C \geq 0$. For each $x \in X$, we choose $\gamma_{x} \in \Gamma$ with $d\left(x, \gamma_{x}\right) \leq C$. Then for any $R>0$ and any $x \in X$, we have

$$
B(x, R) \subset \bigcup_{\gamma \in B(x, R+C) \cap \Gamma} B(\gamma, C) \subset \bigcup_{\gamma \in B\left(\gamma_{x}, R+2 C\right) \cap \Gamma} B(\gamma, C) .
$$

Since $\Gamma$ has bounded geometry,

$$
\sup \left\{\#\left(B\left(\gamma_{x}, R+2 C\right) \cap \Gamma\right) \mid x \in X\right\}<\infty .
$$

It follows from condition (c) that having bounded coarse geometry is a coarse geometric property; that is, for two coarsely equivalent metric spaces $X$ and $Y$, $X$ has bounded coarse geometry if and only if $Y$ does as well. Conditions (b) and (c) are useful when we show that some spaces have bounded coarse geometry. On the other hand we can use condition (d) to prove that some space does not have bounded coarse geometry.

\section{EXAMPLE A.5}

Let $X_{p}$ be a metric space in Example 2.2. Let $k$ be a positive integer. We take the subset $(k \mathbb{Z})^{n}$ of $\ell_{p}(n)$ for $n \in \mathbb{N}$. When we naturally regard $\Gamma_{k}:=\bigsqcup_{n \in \mathbb{N}}(k \mathbb{Z})^{n}$ as a subset of $X_{p}, \Gamma_{k}$ is a $(k-0.01)$-separated net of $X_{p}$. Since $\Gamma_{k}$ does not have bounded geometry, it follows from condition (d) that $X_{p}$ does not have bounded coarse geometry. 
Acknowledgment. The authors would like to thank Professor Hiroyasu Izeki for very helpful discussions.

\section{References}

[1] H. Emerson and R. Meyer, Dualizing the coarse assembly map, J. Inst. Math. Jussieu 5 (2006), 161-186. MR 2225040. DOI 10.1017/S147474800500023X.

[2] T. Fukaya and S. Oguni, Coronae of relatively hyperbolic groups and coarse cohomologies, J. Topol. Anal., published electronically September 1, 2015. DOI 10.1142/S1793525316500151.

[3] N. Higson and J. Roe, "On the coarse Baum-Connes conjecture" in Novikov Conjectures, Index Theorems and Rigidity, Vol. 2 (Oberwolfach, 1993), London Math. Soc. Lecture Note Ser. 227, Cambridge Univ. Press, Cambridge, 1995, 227-254. MR 1388312. DOI 10.1017/CBO9780511629365.008.

[4] - Analytic K-homology, Oxford Math. Monogr., Oxford Univ. Press, Oxford, 2000. MR 1817560.

[5] P. K. Hotchkiss, The boundary of a Busemann space, Proc. Amer. Math. Soc. 125 (1997), 1903-1912. MR 1425125. DOI 10.1090/S0002-9939-97-04166-X.

[6] A. Papadopoulos, Metric Spaces, Convexity and Nonpositive Curvature, IRMA Lect. Math. Theor. Phys. 6, Eur. Math. Soc., Zürich, 2005. MR 2132506.

[7] J. Roe, Coarse Cohomology and Index Theory on Complete Riemannian Manifolds, Mem. Amer. Math. Soc. 104, Amer. Math. Soc., Providence, 1993. MR 1147350. DOI 10.1090/memo/0497.

[8] Lectures on Coarse Geometry, Univ. Lecture Ser. 31, Amer. Math. Soc., Providence, 2003. MR 2007488. DOI 10.1090/ulect/031.

[9] R. Willett, Band-dominated operators and the stable Higson corona, Ph.D. dissertation, Pennsylvania State University, State College, Penn., 2009. MR 2713972.

[10] Some 'homological' properties of the stable Higson corona, J. Noncommut. Geom. 7 (2013), 203-220. MR 3032816. DOI $10.4171 / \mathrm{JNCG} / 114$.

[11] G. Yu, Coarse Baum-Connes conjecture, K-Theory 9 (1995), 199-221. MR 1344138. DOI 10.1007/BF00961664.

[12] - The coarse Baum-Connes conjecture for spaces which admit a uniform embedding into Hilbert space, Invent. Math. 139 (2000), 201-240. MR 1728880. DOI $10.1007 / \mathrm{s} 002229900032$.

Fukaya: Mathematical Institute, Tohoku University, Sendai, Japan; tomo@math.tohoku.ac.jp

Oguni: Department of Mathematics, Faculty of Science, Ehime University, Matsuyama, Japan; oguni@math.sci.ehime-u.ac.jp 